\title{
Pricing of pharmaceuticals
}

Ithough the pricing of

pharmaceuticals may be considered to be rather confusing, it is important to understand that this depends on several factors:

\section{Drug development costs}

It is estimated that it costs 1 billion US dollars to bring a drug to market, with the estimate rising to 4 billion US dollars if we take into consideration the cost of the drugs that fail during the premarketing phases and thus never make it to the market.

The availability of drugs in various markets

Although the cost of drug development is a finite cost, the ability of companies to recover the cost in various markets varies. Those countries which introduce the drugs soon after they gain a marketing authorization may be in a better position to get reduced prices as the pharmaceutical companies have a longer time to recover the expenses. In the most recent Patient's WAIT (Patients Waiting to Access Innovative Therapies) benchmarking analysis, which was conducted in 14 European countries, the duration for the introduction of 114 products ranged $88-392$ days. As for the number of drugs introduced in these counties, the availability ranged 39 $86 \%$. Malta was not one of the countries that were surveyed. Unfortunately in Malta the reimbursement of innovative drugs is very much delayed; in some cases the drug is only introduced on the government list once the patent has expired. Thus to make the innovative drugs available to the patients in Malta many companies launch the product on the private market. However the uptake is usually very small (it is estimated that $<1 \%$ of patients opt to purchase drugs for chronic diseases, rather than take what is given for free). The low volume of sales is reflected in the price and usually this is higher than in those countries where the product is part-financed by the government.

The government purchases the drugs that are distributed through the POYC,

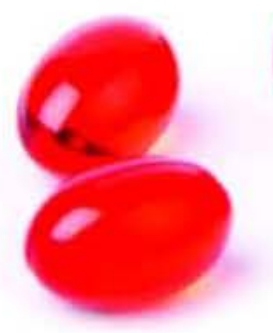

by tender. Once the patent expires, the price of the generic versions of the drugs are a fraction of the price of the originator. In most health systems the money saved from purchasing generic drugs is reinvested in purchasing innovative drugs. PRIMA is encouraging that local health authorities do the same.

\section{Size of population}

Orphan drugs are obviously going to be more expensive, since in many instances expensive specialized technologies are used to develop the drugs, which ultimately result in a limited amount of revenue.

\section{Payment of drugs}

In most EU countries there is a holistic system whereby drugs bought in private retail pharmacies are included in a reimbursement system. In Malta the system is different since there are two separate markets: the private market where the patient pays the full price of the drugs, and the public market which is wholly financed by the government.

\section{Pricing in different countries}

To make sure that the innovative drugs can be afforded by all countries, pharmaceutical companies do not set a fixed price. Prices in the US and Japan are usually more expensive than in European countries. There are also discrepancies in Europe. This 'tiered pricing' is however creating problems, example, countries such as Spain and Italy have implemented price cuts to meet the strict TROIKA criteria in order to be eligible for financial aid from the EU. This is leading to a parallel trade out of these countries, which in turn is causing drug shortages in these countries. Although 'free movement of goods' has been advocated by the EU for the past 50 years, the pharmaceutical industry is asking that parallel trade is temporarily suspended until the financial crisis is under control.

Duration of patent and data exclusivity

The duration of a patent for chemical entities is 10 years and this starts to expire as soon as the chemical entity is registered. If one eliminates the development period, the remaining period is usually $<6$ years. The cost of development has to be recovered during this time.

\section{Reference pricing}

This is the practice whereby the health authorities of countries compare the prices in other countries to establish a price that they are willing to pay for the purchase of the drug. For drugs that are given for free, the Maltese government compares the prices in a set of countries which have a GDP $+1-20 \%$ with respect to the Maltese GDP.

\section{Technology used to produce the drugs}

Today research is moving away from solid dosage forms and moving towards the development of biological treatments. These treatments are generally derived from natural products and are more costly to produce. They usually have to go through many processes to purify the product, and capacity of production is limited. $S$ 